\title{
APPLICATION OF DECOMPOSITION TO HYPERBOLIC, PARABOLIC, AND ELLIPTIC PARTIAL DIFFERENTIAL EQUATIONS
}

\author{
G. ADOMIAN \\ Center for Applied Mathematics \\ University of Georgia \\ Athens, Georgia 30602 \\ (Received September 22, 1987)
}

\begin{abstract}
The decomposition method is applied to examples of hyperbolic, parabolic, and elliptic partial differential equations without use of linearization techniques. We consider first a nonlinear dissipative wave equation; second, a nonlinear equation modeling convection-diffusion processes; and finally, an elliptic partial differential equation.
\end{abstract}

KEY WORDS AND PHRASES. Decomposition method, linear and nonlinear partial differential equations, parabolic equations, elliptic equations, hyperbolic equations. 1980 AMS SUBJECT CLASSIFICATION CODES. 35L35, 35L70.

1. INTRODUCTION :

The decomposition method [1] has developed rapidly and is now providing solutions in the form of converging analytic series for broad categories of ordinary or partial differential equations or systems of equations with given initial/boundary conditions. An important advantage is that linearization is not required. The rather global character is shown by application to some typical examples - a dissipative wave equation, and equation modeling convection-diffusion processes, and an elliptic equation without the use of Iinearization techniques.

2. HYPERBOLIC CASE :

Consider the dissipative wave equation $u_{t t}-u_{x x}+(\partial / \partial t) f(u)=0$ with $f(u(x, t))$ a continuous bounded function and $(t, x) \varepsilon[0, T] \times R$. Let $L_{t}=\partial^{2} / \partial t^{2}$ and $L_{x}=\partial^{2} / \partial x^{2}$ and write

$$
L_{t} u-L_{x} u=-(\partial / \partial t) f(u(x, t))
$$


Using the decomposition method [1] we solve for each linear term thus

$$
\begin{aligned}
& L_{t} u=L_{x} u-(\partial / \partial t) f(u) \\
& L_{x} u=L_{t} u+(\partial / \partial t) f(u)
\end{aligned}
$$

Operating with the inverses, we have $L_{t}^{-1} L_{t} u=u-\phi_{t}$ and $L_{x}^{-1} L_{x} u=u-\phi_{x}$, where the homogeneous solutions are evaluated from the given initial/boundary conditions. Thus (2.2) becomes

$$
\begin{aligned}
& u=\phi_{t}+L_{t}^{-1} L_{x} u-L_{t}^{-1}(\partial / \partial t) f(u) \\
& u=\phi_{x}+L_{x}^{-1} L_{t} u+L_{x}^{-1}(\partial / \partial t) f(u)
\end{aligned}
$$

Adding and dividing by two

$$
\begin{aligned}
u= & (1 / 2)\left(\phi_{t}+\phi_{x}\right)+(1 / 2)\left(L_{t}^{-1} L_{x}+L_{x}^{-1} L_{t}\right) u \\
& -(1 / 2)\left(L_{t}^{-1}-L_{x}^{-1}\right)(\partial / \partial t) f(u)
\end{aligned}
$$

or $i f$

$$
\begin{aligned}
& K=(1 / 2)\left(L_{t}^{-1} L_{x}+L_{x}^{-1} L_{t}\right) \\
& G=-(1 / 2)\left(L_{t}^{-1}-L_{x}^{-1}\right) \\
& u_{0}=(1 / 2)\left(\phi_{t}+\phi_{x}\right)
\end{aligned}
$$

we have

$$
u=u_{0}+K u+G(\partial / \partial t) f(u)
$$

a result also obtained by operating on (2.1) with $\left(L_{t}^{-1}-L_{x}^{-1}\right)$.

Let $u=\sum_{n=0}^{\infty} u_{n}$ with $u_{0}$ as defined and let $f(u)=\sum_{n=0}^{\infty} A_{n}$, where the $A_{n}$ are generated for the specific function $f(u)$ as discussed in [1]. Now

$$
u=u_{0}+k \sum_{n=0}^{\infty} u_{n}+G(\partial / \partial t) \sum_{n=0}^{\infty} A_{n}
$$

We define

$$
u_{n+1}=K u_{n}+G(\partial / \partial t) A_{n}
$$



for $n>0$ to complete the solution. An n-term approximation $\phi_{n}=\sum_{1=0}^{n-1} u_{1}$ suffices
as discussed in $[1,2]$.

\section{PARABOLIC CASE:}

Consider a nonlinear parabolic partial differential equation modeling convectiondiffusion processes given in the form

$$
u_{t}-u_{x x}+f(u, t, x) u_{x}=0
$$

on a defined finite region on $R$ with $t>0$. Assume $\alpha$ is a constant, $f$ is a smooth function of $t, x, u$, and the initial/boundary conditions are given. of course, to obtain a quantitative solution, the specific form of $f$ is required. When $1 t 1 s$, any separable terms in $x$ and $t$ will be designated by $-g(x, t)$ and any remaining term dependent on $u$, and multiplying $u_{x}$ can be written $N\left(u, u_{x}\right)$. Let $L_{t}=\partial / \partial t$ and $L_{x}=\partial^{2} / \partial x^{2}$ and write $(2.1)$ as

$$
L_{t} u-\alpha L_{x} u=g-N\left(u_{,} u_{x}\right)
$$

The decomposition method solves for each linear operator term in turn; thus

$$
\begin{aligned}
& L_{t} u=g+\alpha L_{x} u-N\left(u, u_{x}\right) \\
& L_{x} u=-\alpha^{-1} g+\alpha^{-1} L_{t} u+\alpha^{-1} N\left(u, u_{x}\right)
\end{aligned}
$$

Since $L_{t}^{-1} L_{t} u=u-A=u-u(x, 0)$ and $L_{x}^{-1} L_{x} u=u-B-C x$, we obtain

$$
\begin{aligned}
& u=A+L_{t}^{-1} g+\alpha L_{t}^{-1} L_{x} u-L_{t}^{-1} N\left(u, u_{x}\right) \\
& u=B+C x-\alpha^{-1} L_{x}^{-1} g+\alpha^{-1} L_{x}^{-1} L_{t} u+\alpha^{-1} L_{x}^{-1} N\left(u, u_{x}\right)
\end{aligned}
$$

$A$ is the initial condition, $B$ and $C$ are evaluated from the remaining two conditions. We add the two equations for $u$ and divide by two, obtaining a single equation for u. If we define

$$
\begin{aligned}
& u_{0}=(1 / 2)\left\{u(x, 0)+B+C x+L_{t}^{-1} g-\alpha^{-1} L_{x}^{-1} g\right\} \\
& K=-(1 / 2)\left\{\alpha L_{t}^{-1} L_{x}+\alpha^{-1} L_{x}^{-1} L_{t}\right\} \\
& G=-(1 / 2)\left\{L_{t}^{-1}-\alpha^{-1} L_{x}^{-1}\right\}
\end{aligned}
$$

we have

$$
u=u_{0}+K u+G \cdot N\left(u, u_{x}\right)
$$



The solution by decomposition is $u=\sum_{n=0}^{\infty} u_{n}$ with the given $u_{0}$ and the remaining
components given by

$$
u_{n+1}=K u_{n}+G A_{n}
$$

for $n>0$, where the $A_{n}$ are defined [1] for $N\left(u, u_{x}\right)$ with $N\left(u, u_{x}\right)=\sum_{n=0}^{\infty} A_{n}$. Thus

$$
u=\sum_{n=0}^{\infty} k^{n} u_{0}+G \cdot N u
$$

is the complete solution since the $A_{n}$ are easily evaluated for any specific function f. A practical solution is given by $\phi_{n}=\sum_{i=0}^{n-1} u_{1}$ since the solution converges
generally quite rapidly.

The solution can also be made by operating on (2.2) with $\left(L_{t}^{-1}-\alpha^{-1} L_{x}^{-1}\right)$. Let us designate the solution of $L_{t} u=0$ by $\phi_{t}$ and of $L_{x} u=0$ by $\phi_{x}$, 1.e., $L_{t}^{-1} L_{t} u=u-\phi_{t}$ and $L_{x}^{-1} L_{x} u=u-\phi_{x} \cdot$ We obtain

$$
\begin{aligned}
u-\phi_{t}-\alpha L_{t}^{-1} L_{x} u-\alpha^{-1} L_{x}-1 L_{t} u+u-\phi_{x} \\
=\left(L_{t}^{-1}-\alpha^{-1} L_{x}^{-1}\right)\left(g-N\left(u, u_{x}\right)\right) \\
u=(1 / 2)\left(\phi_{t}+\phi_{x}\right)+(1 / 2)\left(L_{t}^{-1}-\alpha^{-1} L_{x}^{-1}\right) g \\
-(1 / 2)\left(\alpha L_{t}^{-1} L_{x}+\alpha^{-1} L_{x}^{-1} L_{t}\right) u \\
-(1 / 2)\left(L_{t}^{-1}-\alpha^{-1} L_{x}^{-1}\right) N\left(u, u_{x}\right)
\end{aligned}
$$

or

$$
u=u_{0}+K u+G \cdot N\left(u, u_{x}\right)
$$

Though the result is the same and $\phi_{t}$, $\phi_{x}$ are evaluated from the given conditions, writing $u_{0}$ as

$$
u_{0}=(1 / 2)\left(\phi_{t}+\phi_{x}\right)+(1 / 2)\left(L_{t}^{-1}-\alpha^{-1} L_{x}^{-1}\right) g
$$

means the result is not limited to the given parabolic equation - the derivatives can be of any order. The nonlinear term $N\left(u, u_{x}\right)$ can also be more general. The $A_{n}$ can be generated for composite nonlinear functions [1]. 


\section{ELLIPTIC CASE:}

The elliptic equation $\nabla^{2} u+k(x, y, z) u=f(x, y, z)$ arises in some problems of physics and engineering. Let's consider the case:

$$
\begin{aligned}
& u_{x x}+u_{y y}+k(x, y) u=0 \\
& k(x, y)=x^{2}+y^{2}
\end{aligned}
$$

Define $L_{x}=\partial^{2} / \partial x^{2}, L_{y}=\partial^{2} / \partial y^{2}$ and write

$$
\left[L_{x}+L_{y}\right] u+k u=0
$$

Solve for each linear operator term in turn. Then

$$
\begin{aligned}
& L_{x} u=-k u-L_{y} u \\
& L_{y} u=-k u-L_{x} u
\end{aligned}
$$

Apply the inverses $\mathrm{L}_{x}^{-1}$ to the first, $\mathrm{L}_{\mathrm{y}}^{-1}$ to the second. Then since $L_{x}^{-1} L_{x} u=u-\phi_{x}, L_{y}^{-1} L_{y} u=u-\phi_{y}$, where $\phi_{x}, \phi_{y}$ are defined by the initial/boundary conditions, we have

$$
\begin{aligned}
& u=\phi_{x}-L_{x}^{-1}\left[x^{2}+y^{2}\right] u \\
& u=\phi_{y}-L_{y}^{-1}\left[x^{2}+y^{2}\right] u
\end{aligned}
$$

Choosing convenient conditions $u=0$ at $x=0$ or $y=0$ and $u=\sin y$ at $x=1$ and $\sin x$ at $y=1$, we have

$$
\begin{aligned}
& \phi_{x}=A+B x=x \sin y \\
& \phi_{y}=C+D y=y \sin x
\end{aligned}
$$

Let $\phi_{x}$ and $\phi_{y}$ represent the $u_{0}$ term of the decomposition $u=\sum_{n=0}^{\infty} u_{n}$ in the two equations for $u$ in $(4.3 a, b)$. Thus, considering both in parallel

$$
\begin{aligned}
& u_{0}=x \sin y \\
& u_{0}=y \sin x
\end{aligned}
$$


so that

$$
\begin{aligned}
& u=u_{0}-L_{x}^{-1}\left(x^{2}+y^{2}\right) \sum_{n=0}^{\infty} u_{n} \\
& u=u_{0}-L_{y}^{-1}\left(x^{2}+y^{2}\right) \sum_{n=0}^{\infty} u_{n}
\end{aligned}
$$

Thus

$$
\begin{aligned}
& u_{n+1}=-L_{x}^{-1}\left(x^{2}+y^{2}\right) u_{n} \\
& u_{n+1}=-L_{y}^{-1}\left(x^{2}+y^{2}\right) u_{n}
\end{aligned}
$$

for $n \geq 0$ where $u_{1}$ for $(4.5 a)$ uses (4.4a), etc. For example,

$$
\begin{aligned}
& u_{1}=-L_{x}^{-1}\left(x^{2}+y^{2}\right) u_{0} \\
& u_{1}=-L_{y}^{-1}\left(x^{2}+y^{2}\right) u_{0}
\end{aligned}
$$

The one-term approximation to $u$ is given by $\Phi_{1}=(x \sin y+y \sin x) / 2$. We see using the first terms of the trigonometric series that $\Phi_{1}=x y$. We observe from the $u_{1}$ term and the second term of the expansion for $\sin y$, we get $-x^{3} y^{3} / 3$ ! .

From the $u_{2}$ term $-L_{x}^{-1} L_{y}\left[x^{3} / 3\right.$ !)sin $\left.y\right]$ and the third term of sin $y$, we get $x^{5} y^{5} / 5$ !, etc. The n-term approximant $\Phi_{n}$ is given by the $n$-term series for sin $x y$ plus noise terms. To save computation, we can substitute $u=s i n$ and verify it is indeed the correct solution. If we calculate several terms, it is easy to see cancellation of terms other than the series for sin $x y$. Or, if analytic nonlinearities $f(u)$ are involved, the appropriate $A_{n}$ polynomials are generated and we

let $f(u)=\sum_{n=0}^{\infty} A_{n}$

To make some checks of accuracy of the methodology, we consider the onedimensional case $d^{2} u / d x^{2}-40 x u=2$ with $u(-1)=u(1)=0$. Here $L=d^{2} / d x^{2}$ and we have $L x=2+40 x u$. This is a relatively stiff case because of the large coefficient of $u$, and the nonzero forcing function yields an additional Airy-like function. Operating with $L^{-1}$ yields $u=A+B x+L^{-1}(2)+L^{-1}(40 x u)$. Let $u_{0}=A+B x+$ $L^{-1}(2)=A+B x+x^{2}$ and let $u=\sum_{n=0}^{\infty} u_{n}$ with the components to be determined so
that

the sum is $u$. We identify $u_{n+1}=L^{-1}\left(40 x_{n}\right)$. Then all components can be 
determined, e.g., $u_{1}=(20 / 3) A x^{3}+(10 / 3) B x^{4}+2 x^{5}$ and $u_{2}=(80 / 9) A x^{6}+(200 / 63) B x^{7}$ $+(10 / 7) x^{8}$, etc. An n-term approximant $\phi_{n}=\sum_{i=0}^{n-1} u_{1}$ with $n=12$ for $x=0.21 s$ given by -0.135649 , for $x=0.4$ is given by -0.113969 , for $x=0.6$ is given by -0.083321 , for $x=0.8$ is given by -0.050944 , and for $x=1.0$ is, of course, zero. These easily-obtained results are correct to seven digits. We see that a better solution is obtained and much more easily than by variational methods. The solution is found just as easily for nonlinear versions without linearization.

REFERENCES

1. ADOMIAN, G., Nonlinear Stochastic Operator Equations, Academic Press, (1986).

2. ADOMIAN, G., Applications of the Decomposition Method to the Navier-Stokes Equations, J. Math. Ana 1. and Applic., 119, 1 and 2, (1986).

3. ADOMIAN, G., Decomposition Solution for Duffing and Van der Pol Oscillators, Int. J. of Math. Sciences, 9 , 4, (1986), 731-2.

4. Bellomo, N. and RIGANTI, R., Semilinear Stochastic Systems: Analysis With the Method of the Stochastic Green's Function and Application in Mechanics, J. Math. Anal. and Applic. 96, 2, (1983).

5. BELlomo, N. and MONACO R., A Comparison Between Decomposition Methods and Perturbation Techniques for Nonlinear Random Differential Equations, J. Math. Ana 1. adn Applic., 110, 2, (1985).

6. BONZANI, I., On a Class of Nonlinear Stochastic Dynamical Systems: Analysis of the Transient Behavior, J. Math. Anal. and Applic., in press.

7. RACH, R., A Convenient Computational Form for the A Polynomials, J. Math. Anal. and Applic., 102 2, (1984), 415-419.

8. GABETTA, E., On a Class of Semilinear Stochastic Systems in Mechanics with Quadratic Type Nonlinearities: Time Evolution of the Probability Density, J. Math. Anal. and Applic., in press.

9. RACH, R., On Continuous Approximate Solutions of Nonlinear Differential Equations J. Math. Anal. and Applic., in press.

10. RACH, R., On the Adomian (Decomposition) Method and Comparisons with Picard's Method, J. Math. Anal. and Applic., in press.

11. BIGI, D. and RIGANTI, R., Solutions of Boundary-Value Problems by Decompoistion Me thod.

12. BONZANI, I., Analysis of Stochastic Van der Pol Oscillators Using the Decomposition Method, Complex and Distributed Systems: Analysis, Simulation, and Control, eds. S. C. Tzafestas and P. Borne, Elsevier, (1986).

13. RIGANTI, R., Analytical Study of the Class of Nonlinear Stochastic Autonomous Oscillators with One Degree of Freedom, Meccanica, 14, 4, (1979), 180-86.

14. BELlOMO, N., and RIGANTI, R., Nonlinear Stochastic Systems in Physics and Mechanics, World Scientific Publishing Co., Singapore, (1987).

15. ADOMIAN, G., Applications of Nonlinear Stochastic Systems Theory to Physics, Reide1, Dordrecht, (1988). 


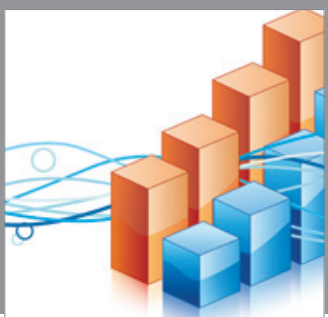

Advances in

Operations Research

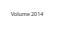

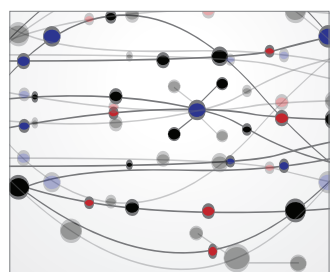

\section{The Scientific} World Journal
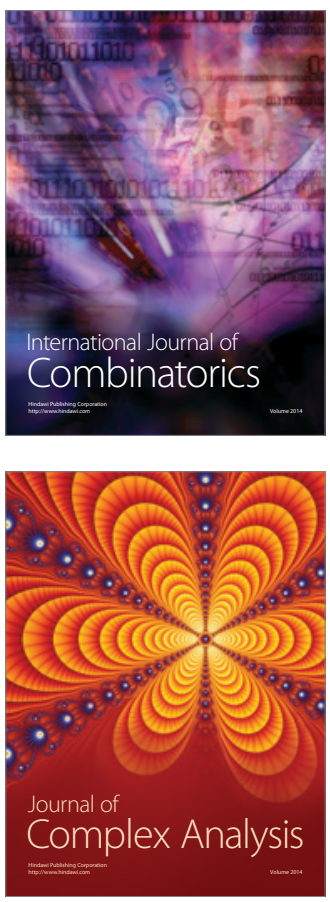

International Journal of

Mathematics and

Mathematical

Sciences
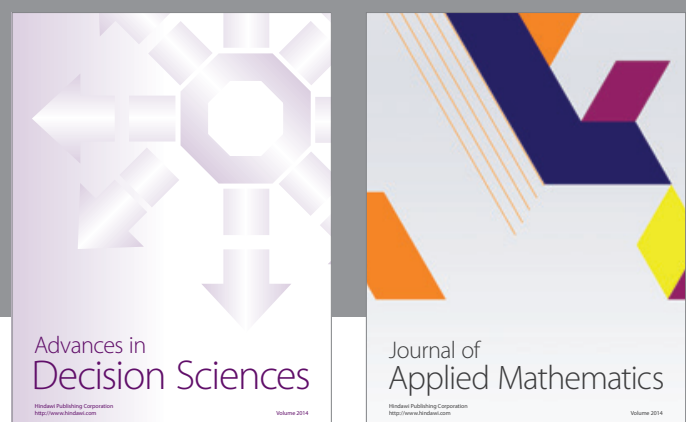

Journal of

Applied Mathematics
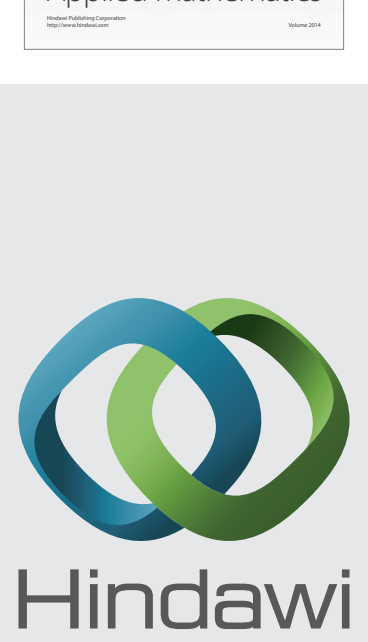

Submit your manuscripts at http://www.hindawi.com
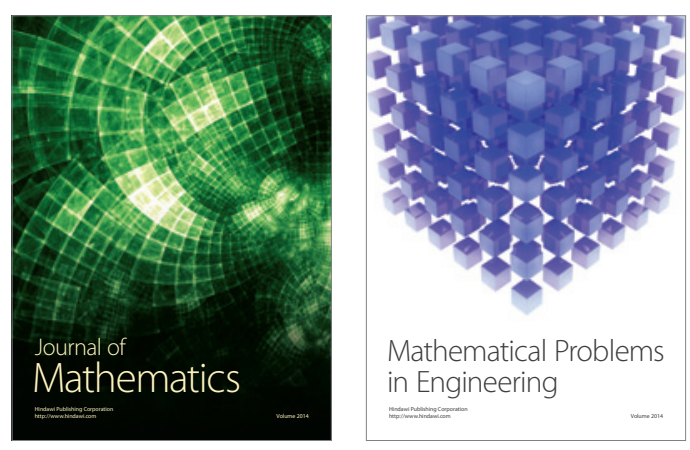

Mathematical Problems in Engineering
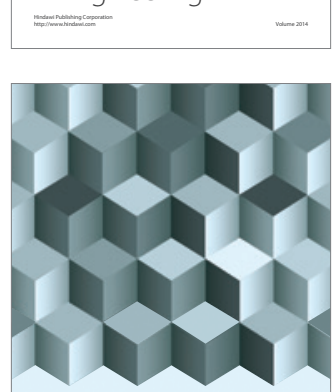

Journal of

Function Spaces
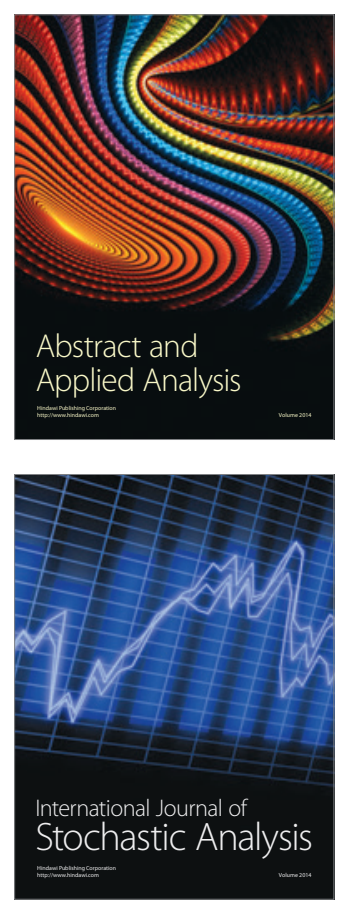

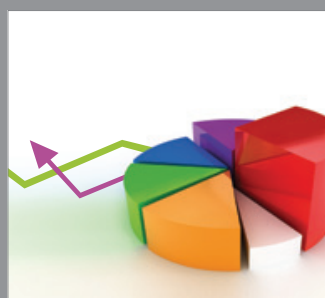

ournal of

Probability and Statistics

Promensencen
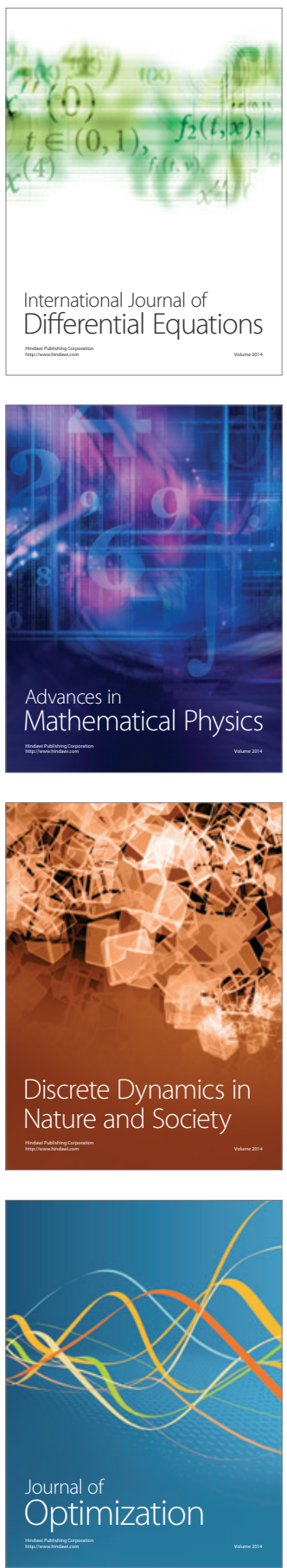